\title{
Microbial community structure and function of Nitrobenzene reduction biocathode in response to carbon source switchover
}

\author{
Bin Liang ${ }^{a}$, Haoyi Cheng ${ }^{b}$, Joy D. Van Nostrand ${ }^{c}$, Jincai $\mathrm{Ma}^{c}, \mathrm{Hao} \mathrm{Yu}^{a}$, \\ Deyong Kong ${ }^{a}$, Wenzong Liu ${ }^{b}$, Nanqi Ren ${ }^{a}$, Liyou $\mathrm{Wu}^{c}$, Aijie Wang ${ }^{a, b, *}$, \\ Duu-Jong Lee ${ }^{a, d, *}$, Jizhong Zhou ${ }^{c, e, f}$ \\ a State Key Laboratory of Urban Water Resource and Environment, Harbin Institute of Technology, Harbin 150090, \\ PR China \\ ${ }^{\mathrm{b}}$ Research Center for Eco-Environmental Sciences, Chinese Academy of Sciences, Beijing 100085, PR China \\ c Institute for Environmental Genomics and Department of Microbiology and Plant Biology, University of Oklahoma, \\ Norman, OK 73019, USA \\ ${ }^{\mathrm{d}}$ Department of Chemical Engineering, National Taiwan University, Taipei 10617, Taiwan \\ e State Key Joint Laboratory of Environment Simulation and Pollution Control, School of Environment, Tsinghua \\ University, Beijing 100084, PR China \\ ${ }^{\mathrm{f}}$ Earth Sciences Division, Lawrence Berkeley National Laboratory, Berkeley, CA 94720, USA
}

\section{A R T I C L E I N F O}

Article history:

Received 29 September 2013

Received in revised form

23 January 2014

Accepted 24 January 2014

Available online 5 February 2014

Keywords:

Biocathode

Nitrobenzene reduction

Microbial community structure

Function

Carbon source switchover

\begin{abstract}
A B S T R A C T
The stress of poised cathode potential condition and carbon source switchover for functional biocathode microbial community influences is poorly understood. Using highthroughput functional gene array (GeoChip v4.2) and Illumina 16S rRNA gene MiSeq sequencing, we investigated the phylogenetic and functional microbial community of the initial inoculum and biocathode for bioelectrochemical reduction of nitrobenzene to less toxic aniline in response to carbon source switchover (from organic glucose to inorganic bicarbonate). Selective transformation of nitrobenzene to aniline maintained in the bicarbonate fed biocathode although nitrobenzene reduction rate and aniline formation rate were significantly decreased compared to those of the glucose-fed biocathode. When the electrical circuit of the glucose-fed biocathode was disconnected, both rates of nitrobenzene reduction and of aniline formation were markedly decreased, confirming the essential role of an applied electric field for the enhancement of nitrobenzene reduction. The stress of poised cathode potential condition led to clear succession of microbial communities from the initial inoculum to biocathode and the carbon source switchover obviously changed the microbial community structure of biocathode. Most of the dominant genera were capable of reducing nitroaromatics to the corresponding aromatic amines regardless of the performance mode. Heterotrophic Enterococcus was dominant in the glucose-fed biocathode while autotrophic Paracoccus and Variovorax were dominant in the bicarbonate-fed biocathode. Relatively higher intensity of diverse multi-heme cytochrome c (putatively involved in electrons transfer) and carbon fixation genes was observed in the
\end{abstract}

\footnotetext{
* Corresponding authors. State Key Laboratory of Urban Water Resource and Environment, Harbin Institute of Technology, Harbin 150090, PR China. Tel./fax: +86 45186282195.

E-mail addresses: waj0578@hit.edu.cn, aijie-team@163.com (A. Wang), djlee@ntu.edu.tw (D.-J. Lee).
} 
biocarbonate-fed biocathode, likely met the requirement of the energy conservation and maintained the nitrobenzene selective reduction capability after carbon source switchover. Extracellular pilin, which are important for biofilm formation and potential conductivity, had a higher gene abundance in the glucose-fed biocathode might explain the enhancement of electro-catalysis activity for nitrobenzene reduction with glucose supply. Dominant nitroaromatics-reducing or electrochemically active bacteria and diverse functional genes related to electrons transfer and nitroaromatics reduction were associated with nitrobenzene reduction efficiency of biocathode communities in response to carbon source switchover.

(c) 2014 Elsevier Ltd. All rights reserved.

\section{Introduction}

Nitrobenzene (NB), a nitroaromatic compound is massively produced and used as an intermediate for manufacturing of dyes, explosives, pesticides, pharmaceuticals, aniline (AN) and solvents (Wang et al., 2011). Significant quantities of NB have been released to the environment, making NB a priority pollutant due to its mutagenicity, carcinogenicity and recalcitrance (Spain, 1995). Some isolated bacteria that can mineralize NB (Ju and Parales, 2010; Ma et al., 2007; MarvinSikkema and de Bont, 1994). However, instead of complete mineralization, most microorganisms are only capable of reducing NB to the less toxic, AN (Marvin-Sikkema and de Bont, 1994; Spain, 1995) via diverse nitroreductases (Roldan et al., 2008). Nitro group reduction is favorable since the electronegativity of the $\mathrm{N}$ atom (+III) can be maintained with the electron donated from $\mathrm{NAD}(\mathrm{P}) \mathrm{H}$, hence this reductive pathway is prevalent in NB-biodegrading strains (Ju and Parales, 2010; Spain, 1995). The reaction pathway for microbial reduction of nitrobenzene (Peres et al., 1998) is shown in Fig. 1.

Bioelectrochemical system (BES) has been developed for enhanced removal of recalcitrant pollutants. Bioelectrochemical reduction of nitroaromatics to the corresponding less toxic aromatic amine products has been extensively studied by biocathode or abiotic cathode BES (Liang et al., 2013; Mu et al., 2009; Shen et al., 2013; Wang et al., 2011). Practical nitroaromatics wastewater varied from the nutrient-rich heterotrophic to the oligotrophic condition, thus it is meaningful for estimating the nitroaromatics removal efficiency and stability with functional biocathode communities upon different carbon sources supply. The microbial community structure in the functional biocathode has been studied using PCR-DGGE, clone libraries, PhyloChip, or highthroughput 454-pyrosequencing technology (Liang et al., 2013; Wang et al., 2011; Wrighton et al., 2010; Zhang et al.,

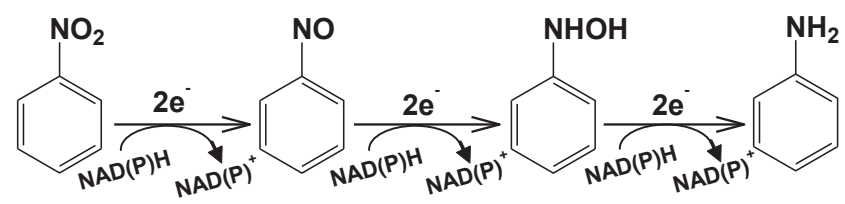

Fig. 1 - The reaction pathway for microbial reduction of nitrobenzene.
2012). Our previous works analyzed nitroaromatics (NB and chloramphenicol) reducing biocathode communities using traditional PCR-DGGE and clone libraries (Liang et al., 2013; Wang et al., 2011), which only provided relatively fewer microbial communities information. Moreover, the clear microbial communities succession from the initial NB-reducing inoculum to the NB-reducing biocathode biofilms, and microbial functional gene diversity, especially putative functional genes related to electrons transfer that in response to carbon source switchover (from organic glucose to inorganic bicarbonate) using high-throughput metagenomics technology have not yet been understood. Illumina MiSeq sequencing platform (Caporaso et al., 2012) and GeoChip-based functional gene arrays (He et al., 2012; Liu et al., 2010; Zhang et al., 2013; Zhou et al., 2013) are suitable tools to characterize microbial community structure, function and metabolic potential in NBreducing biocathode biofilms.

This study investigated the kinetics of NB reduction by biocathode communities and characterized both the microbial phylogenetic and functional communities in the initial inoculum and the NB-reducing biocathode biofilms switched from heterotrophic to autotrophic cultivation using comprehensive GeoChip (v4.2) and 16S rRNA gene based highthroughput Illumina MiSeq sequencing platform. The objectives of this study were to (i) determine the impact of carbon source switchover on the catalytic characterization of NBreducing biocathode and (ii) characterize both the microbial phylogenetic and functional communities in the initial inoculum and the NB-reducing biocathode biofilms in response to carbon source switchover. Our results indicated that the continuous electrical field stimulation and carbon source switchover led to obviously shifts of microbial community structure and revealed that how the biocathode communities maintained the NB selective reduction capability under autotrophic cultivation.

\section{Materials and methods \\ 2.1. Bioelectrochemical reactor setup}

The Polycarbonate BES reactors were constructed by assembling two equal-size Lexan plates $\left(7 \times 7 \times 4 \mathrm{~cm}^{3}\right)$ with a cylindrical cavity ( $5 \mathrm{~cm}$ in diameter and $4 \mathrm{~cm}$ in length) and two equal-size Lexan plates $\left(7 \times 7 \times 1 \mathrm{~cm}^{3}\right)$ as outside baffles and 

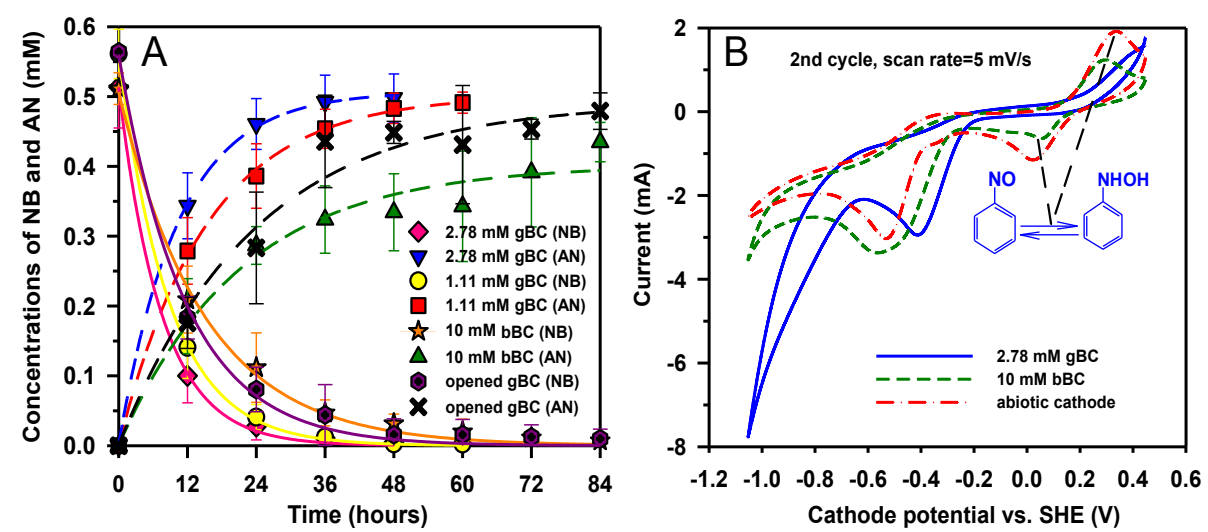

Fig. 2 - NB reduction and AN formation under different operational modes. Opened gBC set (2.78 mM glucose fed) was performed instantly after the gBC test. Lines were fitted with the first-order kinetic model (A). Cyclic voltammograms of the biocathode and abiotic cathode for NB reduction (B).

separated by a cation exchange membrane (Ultrex CMI-7000, Membranes International, USA) (Liang et al., 2013). Graphite fiber brush $(2.5 \mathrm{~cm}$ in diameter and $2.5 \mathrm{~cm}$ in length, TOHO TENAX Co., Ltd., Japan) and carbon cloth $(5 \mathrm{~cm}$ in diameter, non-wet proof, YB-20, YiBang Technology Co. Ltd. China) was used as anode and cathode, respectively. The internal volumes of anode and cathode chambers were both $85 \mathrm{~mL}$. The assembled BES reactors were sterilized at $121^{\circ} \mathrm{C}$ for $30 \mathrm{~min}$. A high-precision resistor $(10 \Omega)$ with the external power $(0.3 \mathrm{~V})$ in series was employed for the connection. A saturated calomel reference electrode (SCE, $0.247 \mathrm{~V}$ vs. standard hydrogen electrode, SHE, model-217, Shanghai Precise. Sci. Instrument Co., Ltd, China) was inserted into the cathode chamber to measure the cathode potential. All of the potential data reported herein were against SHE.

\subsection{Microbial inoculum and BES tests}

The enrichment procedures for NB-reducing microbial consortium and anodic microbial community were described previously (Wang et al., 2011). Activated sludge collected from Taiping Wastewater Treatment Plant (Harbin, China) was used to seed the reactors. NB reduction was tested under four different modes: a) glucose-fed biocathode (gBC; glucose and the electrode serve as intracellular and extracellular electrons donors, respectively), b) biocathode switched from heterotrophic to autotrophic (bicarbonate-fed) cultivation (bBC; electrode was the extracellular electrons donor, no glucose), c) power source and resistor disconnected from the BES (opened biocathode; with only microbial activities and glucose was the intracellular electrons donor), and d) abiotic cathode BES (no microbial inoculation).

The development of the biocathode was carried out (Liang et al., 2013) with enriched NB-reducing consortium $\left(k_{\mathrm{NB}}=0.022 \mathrm{~h}^{-1}\right.$ and $\left.k_{\mathrm{AN}}=0.005 \mathrm{~h}^{-1}\right)$ as the inoculum under the applied voltage of $0.3 \mathrm{~V}$. The initial NB concentration was $0.5 \mathrm{mM}$ in the BES test. Seven biocathode reactors were operated for five fed-batch cycles with $2.78 \mathrm{mM}$ glucose supplied. At the end of the tests, the cathode biofilm of two reactors (NB1\# and NB2\#) were sampled and stored at $-20^{\circ} \mathrm{C}$ before DNA extraction. The remaining five reactors were performed instantly as the open-circuit mode for one cycle after closed biocathode test with the purpose of keeping the same biofilm for the comparison of their efficiency. The glucose worked as the intracellular electron donor for the NB reduction with biofilm communities. The opened biocathode test lasted for $84 \mathrm{~h}$, hence should have minimal effects on the biocathode community structure. These five reactors were operated for another five fed-batch cycles at $1.11 \mathrm{mM}$ glucose and then two reactors (NB4\# and NB7\#) were switched to autotrophic cultivation (10 $\mathrm{mM}$ bicarbonate) for another five fed-batch cycles. One reactor with constant posited abiotic cathode $(-0.5 \mathrm{~V})$ (model-660D, CH Instruments Inc. U.S.) was operated to collect abiotic NB reduction data. Medium composition and preparation of the catholyte and anolyte were described previously (Wang et al., 2011). All tests were conducted at $26 \pm 2{ }^{\circ} \mathrm{C}$.

\subsection{Chemicals and analytical methods}

Nitrosobenzene (>97\% purity), NB and AN (both >99\% purity) and high performance liquid chromatography (HPLC) grade methanol were purchased from Sigma-Aldrich (St. Louis, USA).

NB and metabolites (AN and nitrosobenzene) concentrations for cathode suspension samples were analyzed by a reverse-phase HPLC (model e2695, Waters Co., USA) (Wang et al., 2011).

The electric current (I), NB reduction efficiency $\left(E_{\mathrm{rNB}}, \%\right), \mathrm{NB}$ reduction rate $\left(k_{\mathrm{NB}}\right)$, AN formation rate $\left(k_{\mathrm{AN}}\right)$, AN formation from $\mathrm{NB}\left(\mathrm{E}_{\mathrm{AN}}, \%\right)$ and $\mathrm{NB}$ reduction coulombic efficiency $\left(\mathrm{CE}_{\mathrm{NB}}\right.$ AN, \%) were calculated according to (Wang et al., 2011, 2012). The rates of $\mathrm{NB}$ reduction and $\mathrm{AN}$ formation were fitted using an apparent first-order reaction model: $C=C_{0} e^{-k t}$ and $C=C_{0}(1-$ $\left.\mathrm{e}^{-k t}\right)$, respectively, where $C$ represents the NB or AN concentrations $(\mathrm{mM})$ at time $t(h), C_{0}$ is the initial NB concentration 
Table 1 - The respective $k_{\mathrm{NB}}, k_{\mathrm{AN}}$, linear regression $r^{2}, E_{\mathrm{rNB}-\max }, E_{\mathrm{AN}-\max }$ and $\mathrm{CE}_{\mathrm{NB}-\mathrm{AN}}$ under different operational modes.

\begin{tabular}{|c|c|c|c|c|c|c|c|}
\hline & $k_{\mathrm{NB}}\left(\mathrm{h}^{-1}\right)$ & $k_{\mathrm{AN}}\left(\mathrm{h}^{-1}\right)$ & $r_{\mathrm{NB}}^{2}$ & $r_{\mathrm{AN}}^{2}$ & $E_{\text {rNB-max }} / \%$ & $E_{\mathrm{AN}-\max } / \%$ & $\mathrm{CE}_{\mathrm{NB}-\mathrm{AN}} / \%$ \\
\hline $2.78 \mathrm{mM}$ glucose/biocathode & $0.135 \pm 0.015^{a}$ & $0.094 \pm 0.008^{a}$ & 0.999 & 0.999 & $99.1 \pm 0.7$ & $96.6 \pm 2.5$ & $143.5 \pm 20.1^{a}$ \\
\hline $1.11 \mathrm{mM}$ glucose/biocathode & $0.120 \pm 0.018^{\mathrm{ac}}$ & $0.067 \pm 0.012^{b}$ & 0.999 & 0.999 & $99.6 \pm 0.4$ & $90.7 \pm 1.1$ & $113.0 \pm 39.8^{\mathrm{ab}}$ \\
\hline $10 \mathrm{mM} \mathrm{NaHCO}_{3} /$ biocathode & $0.065 \pm 0.011^{\mathrm{b}}$ & $0.049 \pm 0.004^{c}$ & 0.996 & 0.970 & $99.3 \pm 1.4$ & $90.9 \pm 5.6$ & $82.9 \pm 13.5^{b}$ \\
\hline $2.78 \mathrm{mM}$ glucose/opened biocathode & $0.093 \pm 0.022^{c}$ & $0.050 \pm 0.015^{\mathrm{bc}}$ & 0.996 & 0.975 & $98.3 \pm 2.4$ & $85.0 \pm 4.6$ & - \\
\hline Abiotic cathode $\mathrm{a}^{\mathrm{a}}$ & $0.046 \pm 0.010$ & $0.037 \pm 0.014$ & 0.968 & 0.976 & 88.4 & 64.8 & 57.4 \\
\hline
\end{tabular}

(0.5 mM) or maximum AN production concentration (0.5 mM). The rate constant $k\left(\mathrm{~h}^{-1}\right)$ was calculated using SigmaPlot v11.0. Statistical analysis was performed using SPSS 15.

\subsection{Cyclic voltammetry analysis}

Analysis of cyclic voltammetry (CV) was performed using an electrochemical workstation (model-660D, CH Instruments Inc. U.S.) equipped with three-electrode system. The cathode/ biocathode and the anode of BES were used as the working and counter electrode, respectively. The SCE was the reference electrode and the medium ( $\mathrm{pH} 7.0)$ was the same as the biocathode/abiotic cathode tests (Liang et al., 2013). Concentrations of NB, glucose and bicarbonate, when used, were 0.5 , 2.78 and $10 \mathrm{mM}$, respectively.

\subsection{Illumina MiSeq sequencing, GeoChip hybridization and data analysis}

The details for the DNA extraction, 16S rRNA gene PCR amplification and Illumina MiSeq sequencing, GeoChip hybridization and data analysis are available in the Supplemental Material.

\section{Results and discussion}

\subsection{NB reduction and AN formation by the biocathode communities}

The rates of $\mathrm{NB}$ reduction $\left(r^{2}>0.99\right)$ and $\mathrm{AN}$ formation $\left(r^{2}>0.97\right)$ were fitted with first-order kinetics. The $k_{\mathrm{NB}}$ and $k_{\mathrm{AN}}$ followed: $2.78 \mathrm{mM}$ glucose fed $\mathrm{gBC}>1.11 \mathrm{mM}$ glucose fed $\mathrm{gBC}$ $>2.78 \mathrm{mM}$ glucose fed opened $\mathrm{gBC}>\mathrm{bBC}$. The gBC had significantly larger $k_{\mathrm{NB}}(p<0.01$ or 0.05$)$ and $k_{\mathrm{AN}}(p<0.05$ for both) than either the bBC or opened gBC (Fig. 2A and Table 1). Decrease in glucose concentration in the biocathode BES markedly reduced the $k_{\mathrm{AN}}(p<0.01)$; however, this decrease only significantly lowered the $k_{\mathrm{NB}}(p<0.01)$ for the $\mathrm{bBC}$. The highest maximal $E_{\mathrm{rNB}}\left(E_{\mathrm{rNB}-\mathrm{max}}\right)$ and maximal $E_{\mathrm{AN}}\left(E_{\mathrm{AN}-\mathrm{max}}\right)$ were observed in the $\mathrm{gBC}$ tests at $2.78 \mathrm{mM}$ glucose while the lowest $E_{\text {rNB-max }}$ and $E_{\mathrm{AN} \text {-max }}$ were noted for the opened gBC (Table 1).

The gBC and bBC had similar cathodic potential (approx. $-0.54 \mathrm{~V})$. In $36 \mathrm{~h}$ of testing, the reaction current of $\mathrm{gBC}$ (2.78 mM glucose) decreased to $<0.02 \mathrm{~mA}$, while that of $\mathrm{gBC}$ (1.11 mM glucose) and bBC were maintained at 0.03-0.09 mA. Although the current looks like at same level (Fig. 3), some differences were found among these three modes by calculating the cumulative charge. At initial stage (the first $6 \mathrm{~h}$ for example), more charge was accumulated in $2.78 \mathrm{mM}$ gBC (10.41 C), which was followed by that in $1.11 \mathrm{mM} \mathrm{gBC}(9.25 \mathrm{C})$ and $\mathrm{bBC}(8.36 \mathrm{C})$, indicating the electron flow capability increased with the increasing of glucose. This observation consisted with higher electro-catalytic activity with the present of glucose (Fig. 2B) as well as the higher $k_{\mathrm{NB}}$ with more glucose (Fig. 2A). When the batch test went to the final stage (from 40th to 50th $\mathrm{h}$ for example), the order of cumulative charge turned over ( $0.44 \mathrm{C}$ in $2.78 \mathrm{mM}$ gBC, $1.99 \mathrm{C}$ in $1.11 \mathrm{mM}$ gBC and $2.12 \mathrm{C}$ in $\mathrm{bBC}$ ), which was likely due to low NB concentration (as electron acceptor) limited the electron flow. Here the lower NB concentration left in catholyte caused the lower cumulative charge.

The $\mathrm{CE}_{\mathrm{NB}-\mathrm{AN}}$ of $\mathrm{gBC}(2.78 \mathrm{mM}$ glucose) was significantly higher than that of $\mathrm{bBC}(p<0.001)$ but was only slightly higher than that of $g B C$ (1.11 mM glucose) (Table 1). The preference of glucose as electron donor for NB reduction can be revealed by $\mathrm{CE}_{\mathrm{NB}-\mathrm{AN}} \cdot \mathrm{CE}_{\mathrm{NB}-\mathrm{AN}}$ over $100 \%$ means partial of $\mathrm{NB}$ reduction was not contributed by electrode but by glucose and the higher $\mathrm{CE}_{\mathrm{NB}-\mathrm{AN}}$ represent higher contribution of glucose as electron donor for NB reduction. $C E_{N B-A N}$ increased with adding more glucose in cathode, indicating glucose as electron donor was preferred with higher glucose concentration in catholyte (Table 1). The CV tests showed that NB reduction started at about $-0.2,-0.3$ and $-0.4 \mathrm{~V}$ with gBC (2.78 $\mathrm{mM}$ glucose), bBC

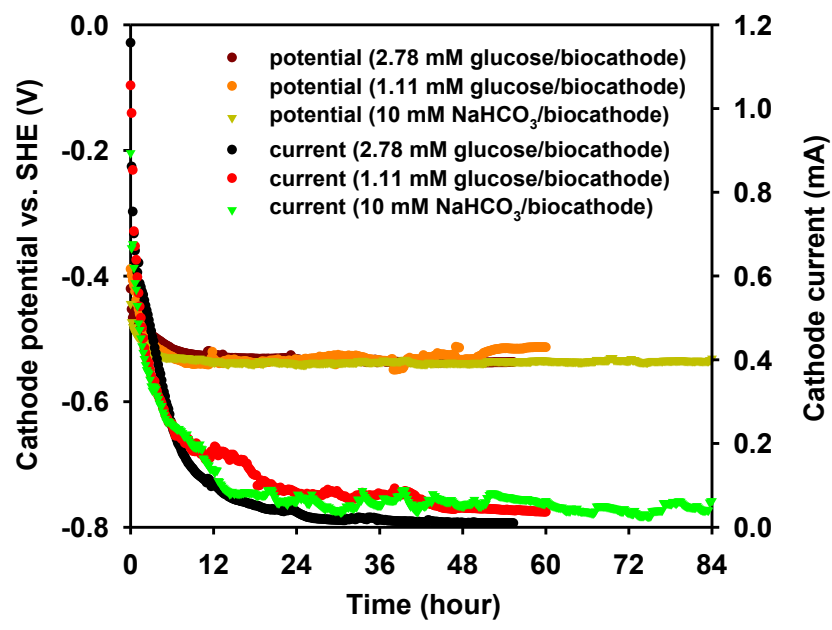

Fig. 3 - Dynamic changes in the cathode potential and current during NB reduction under different operational modes. 
and abiotic cathode, respectively (Fig. 2B), indicating that the biocathode could decrease the over-potentials of nitroaromatic reduction (Liang et al., 2013; Wang et al., 2011). A small quantity of nitrosobenzene (0.02 $\mathrm{mM}$ at 24th h) was noted in the bBC samples but not in the gBC and opened gBC samples. Tests with the abiotic cathode at a constant cathodic potential of $-0.5 \mathrm{~V}$ and initial NB concentration of $0.46 \mathrm{mM}$ yielded an $E_{\mathrm{rNB}}$ of $76.8 \%$, accumulated $0.24 \mathrm{mM}$ nitrosobenzene and formed $0.04 \mathrm{mM}$ AN in $24 \mathrm{~h}$. The performance of the abiotic BES was much poorer than the biocathode BES, correlating with findings in (Wang et al., 2011). The CV analysis also showed that the reversible reaction of phenylhydroxylamine and nitrosobenzene occurred at around 0 and $0.3 \mathrm{~V}$ in the $\mathrm{bBC}$ and abiotic cathode, similar to previous study (Liang et al., 2013). The peak current of the phenylhydroxylamine-nitrosobenzene reversible reaction in the $\mathrm{bBC}$ was lower than in the abiotic cathode (Fig. 2B).

\subsection{Overall microbial phylogenetic and functional gene diversity}

The microbial community diversities and phylogenetic structure of the initial inoculum, $\mathrm{gBC}$ and $\mathrm{bBC}$ were analyzed by Illumina sequencing. Over 20000 sequences were obtained for each sample. Relatively higher OTUs were in the bBC (294 and 251 for two biological replicates) than that of the initial inoculum (239) and gBC (207 and 214 for two biological replicates). The estimated species richness Chao1 for each sample was also consistent with this trend (Fig. 4). Based on the Shannon-Weaver index, the bBC (3.36 and 2.56) also had relatively higher diversity than that of the gBC (2.24 and 2.29). It was interestingly to find that the $\mathrm{bBC}$ communities had relatively higher biodiversity and unique OTUs among three conditions.

The microbial functional gene diversity and metabolic potential of the initial inoculum and biocathode were

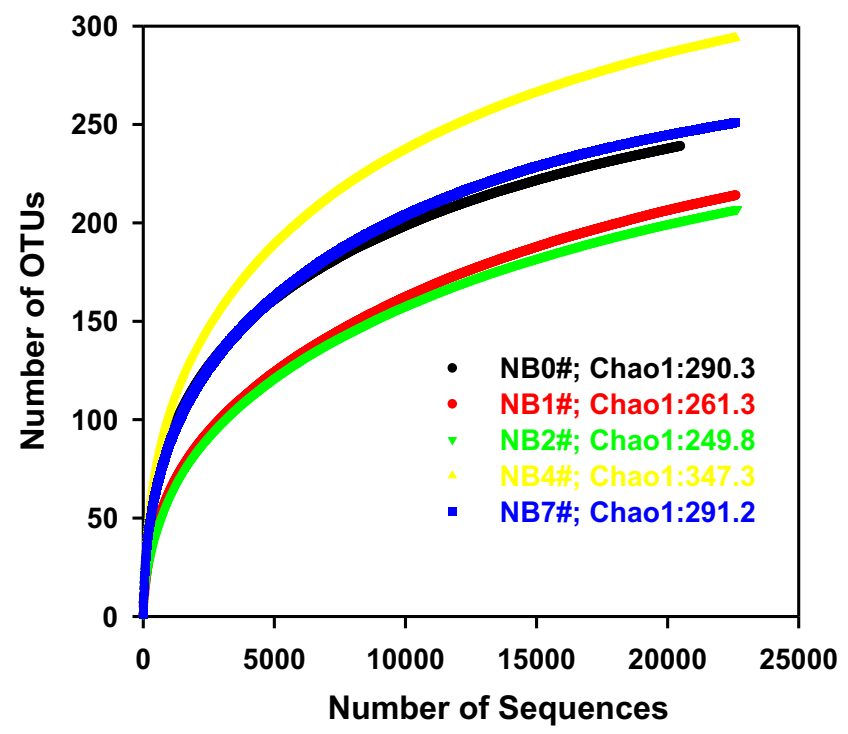

Fig. 4 - Rarefaction curves based on the 16S rRNA gene sequencing of the biocathode (gBC: NB1\# and NB2\#; bBC: NB4\# and NB7\#) and the initial inoculum (NB0\#). The Chao1 was also showed. The OTUs were defined by $97 \%$ cutoff. analyzed with GeoChip (v4.2), which contains 93,902 probes covering 156,719 genes from 738 functional gene families for different microbial functional and biogeochemical processes. A total of 23,339 functional genes were detected in the present samples, of which 6427 functional genes undetected in the initial inoculum but were enriched in the biocathode. Additionally, 1857 functional gene sequences were present in the $\mathrm{bBC}$ but were absent in the gBC, while 1362 were detected in the $g B C$ but were absent in the bBC. Overall, the Shannon-Weiner index and evenness showed little difference among samples although fewer genes were detected in the gBC (NB2\#) (Table S1).

\subsection{Shifts in microbial community structure and composition}

Microbial community structures are highly similar in two biological replicates but different from each other at different modes based on the principal coordination analysis (PCoA) (Fig. 5) and hierarchical clustering analysis of classified OTUs from each sample (Fig. 6A). The total classified OTUs in all three bacterial communities was 488 , thereinto 100 OTUs $(20.49 \%)$ of the total OTUs were shared by them. More than half $(59 \%)$ of the shared OTUs belonged to Proteobacteria and OTUs belonging to Actinobacteria (11\%), Bacteroidetes (14\%) and Firmicutes (13\%) shared at similar levels (Fig. 6B).

A total of 19 phyla were identified and 12, 15 and 16 for the initial inoculum, $\mathrm{gBC}$ and $\mathrm{bBC}$, respectively. Proteobacteria dominated in the bacterial community in the three operational modes. Firmicutes was found to be a dominant group with biocathode mode. However it was sharply decreased (from 40.55 to $7.88 \%$ ) within biocathode communities when switching carbon source from glucose to bicarbonate. The relatively abundance for Proteobacteria (from 43.27 to $68.97 \%$ ) and Bacteroidetes (from 4.95 to $17.88 \%$ ) were increased after

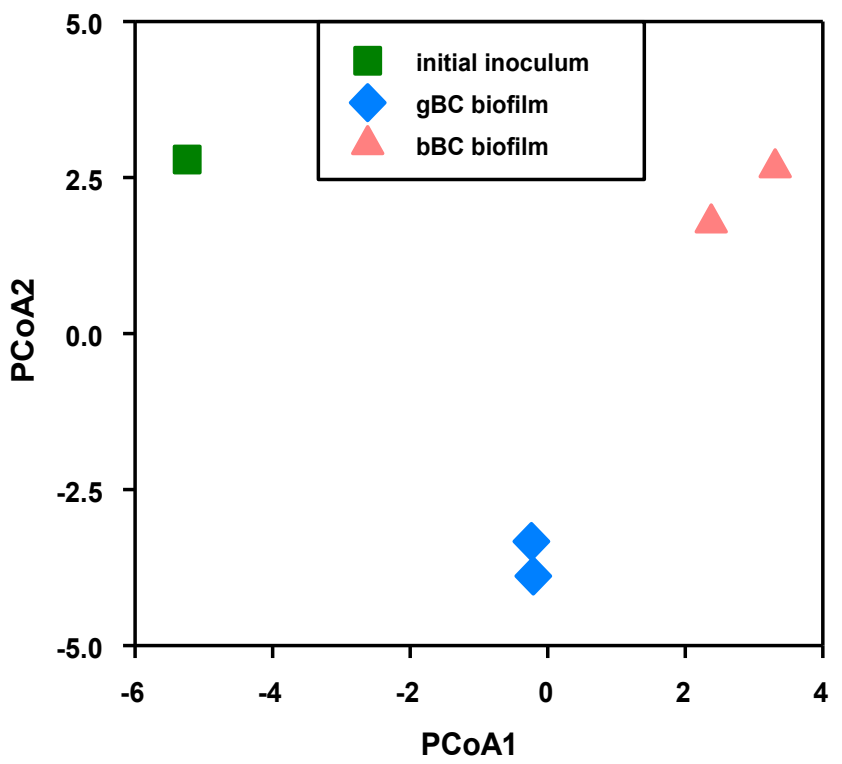

Fig. 5 - Principal coordination analysis (PCoA) of bacterial communities from the initial inoculum, gBC and $b B C$ based on the classified OTUs. 

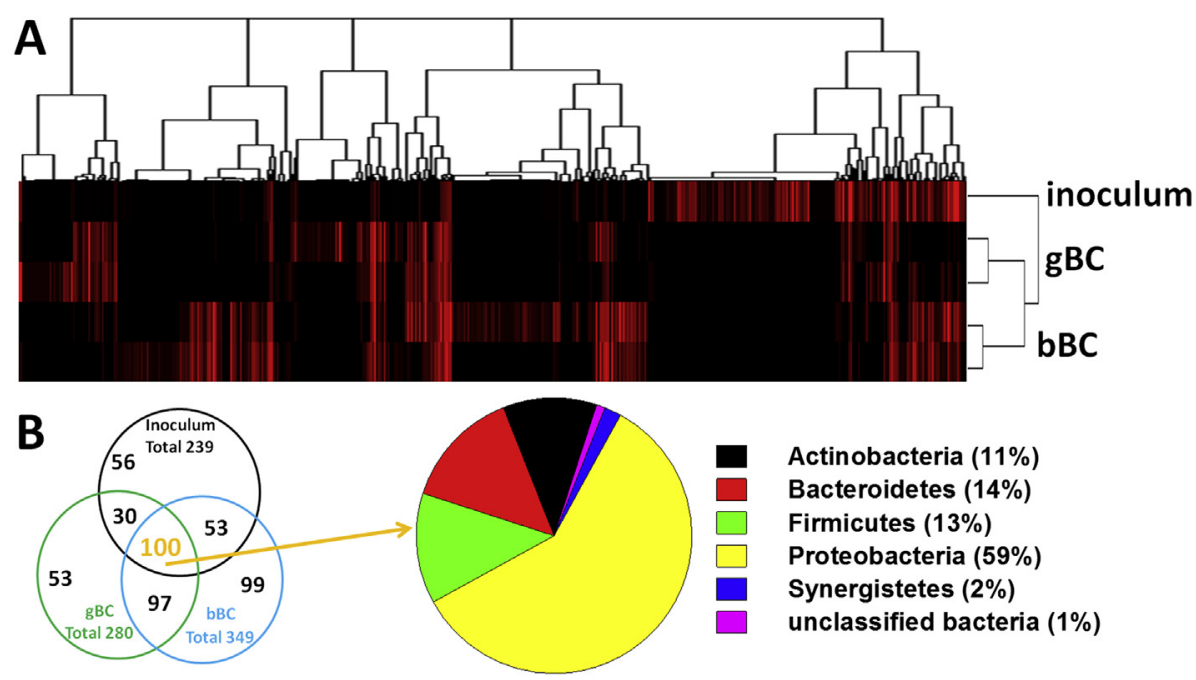

Actinobacteria (11\%)

Bacteroidetes (14\%)

Firmicutes (13\%)

Proteobacteria (59\%)

Synergistetes (2\%)

unclassified bacteria (1\%)
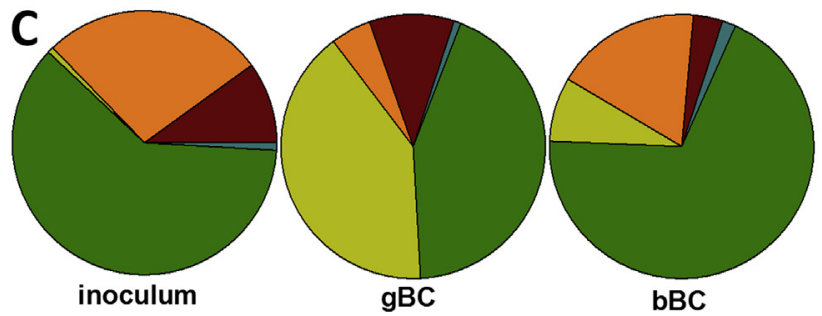

at phylum level

actinobacteria

Bacteroidetes

Firmicutes

Proteobacteria

Others

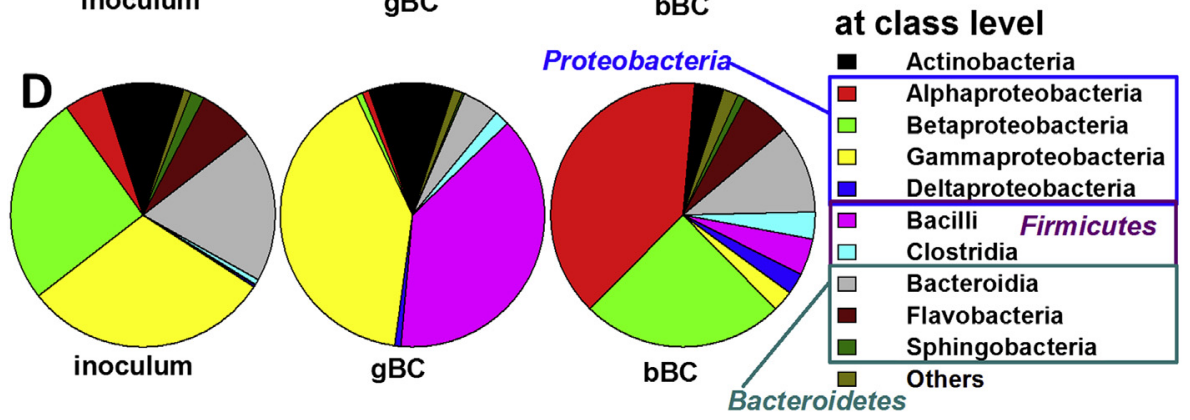

Fig. 6 - Hierarchical cluster analysis of bacterial communities in the initial inoculum, gBC and bBC (A). Overlap of the three bacterial communities from the initial inoculum, gBC and bBC. The shared OTUs (100) were analyzed at phylum levels (B). Taxonomic classification of 16S rRNA gene sequences from bacterial communities of the initial inoculum, gBC and bBC at the phylum (C) and class (D) levels. Relative abundance of each taxon was defined as the percentage of the same taxon to the corresponding total sequences for each sample. Some classes were $<1 \%$ abundances were summarized as others.

carbon source switchover. The gBC (10.39\%) had relatively higher Actinobacteria abundance than that of the bBC $(3.60 \%)$ but was with similar level to the initial inoculum (10.00\%). The initial inoculum (27.22\%) had the highest Bacteroidetes abundance among the three operational modes (Fig. 6C).

A total of 30 classes were classified and the most of the sequences belonged to 10 classes (Fig. $6 \mathrm{D}$ ). Only $0.29-1.42 \%$ of the total sequences among the test samples were unclassified. Bacilli and Gammaproteobacteria species were obviously enriched in the gBC compared to those of the initial inoculum but were both sharply decreased in the bBC after carbon source switchover. Betaproteobacteria species only showed obviously lower abundance in the gBC (only 0.80\%) but Alphaproteobacteria species were only enriched in the $b B C$ (39.00\%) (Fig. 6D). These clear differences strongly indicated that cathode supply electrons mode markedly enriched different bacteria community structure and composition within biocathode compared to that of the initial inoculum and carbon source switchover obviously led to a clear distinction between $\mathrm{gBC}$ and $\mathrm{bBC}$ communities.

\subsection{Potential function of dominant genera}

A total of 179 genera were classified among the test samples. 33 genera with relative abundance $>1 \%$ in the initial inoculum, gBC or bBC communities were showed in the Table 2. Dysgonomonas (17.95\%), Escherichia (17.91\%) and Comamonas (16.74\%) obviously dominated in the initial inoculum. Comamonas was capable of biodegrading NB (Ma et al., 2007). Escherichia, Pseudomonas, Mycobacterium and Flavobacterium were capable of reducing nitroaromatics to corresponding aromatic amines (Marvin-Sikkema and de Bont, 1994). 
Table 2 - Phylogenetic classification of the 165 rRNA gene sequences (relative abundance $>1 \%$ at genus levels) in the initial inoculum, gBC and bBC. Dominant genera in the different operational modes was bolded.

\begin{tabular}{|c|c|c|c|c|c|c|}
\hline Phylum & Class & Family & Genus (\%) & Initial & gBC & $\mathrm{bBC}$ \\
\hline Bacteroidetes & Bacteroidia & Porphyromonadaceae & Dysgonomonas & 17.95 & 0.77 & 0.25 \\
\hline Proteobacteria & $\gamma$-proteobacteria & Enterobacteriaceae & Escherichia/Shigella & 17.91 & 0.64 & 0.03 \\
\hline Proteobacteria & $\beta$-proteobacteria & Comamonadaceae & Comamonas & 16.74 & 0.12 & 2.44 \\
\hline Proteobacteria & $\gamma$-proteobacteria & Pseudomonadaceae & Pseudomonas & 6.40 & 0.29 & 1.72 \\
\hline Actinobacteria & Actinobacteria & Microbacteriaceae & Leucobacter & 5.22 & 0.004 & 0.81 \\
\hline Proteobacteria & $\beta$-proteobacteria & Alcaligenaceae & Achromobacter & 4.00 & 0.25 & 0.92 \\
\hline Actinobacteria & Actinobacteria & Mycobacteriaceae & Mycobacterium & 3.55 & 0.004 & 0.16 \\
\hline Bacteroidetes & Flavobacteria & Flavobacteriaceae & Flavobacterium & 2.73 & 0 & 0 \\
\hline Proteobacteria & $\beta$-proteobacteria & Alcaligenaceae & Bordetella & 2.70 & 0.01 & 0.004 \\
\hline Bacteroidetes & Flavobacteria & Flavobacteriaceae & Myroides & 1.60 & 0 & 0.09 \\
\hline Proteobacteria & $\alpha$-proteobacteria & Xanthobacteraceae & Xanthomonas & 1.44 & 0.004 & 0 \\
\hline Firmicutes & Bacilli & Enterococcaceae & Enterococcus & 0.06 & 38.42 & 4.39 \\
\hline Proteobacteria & $\gamma$-proteobacteria & Moraxellaceae & Psychrobacter & 0 & 18.05 & 0 \\
\hline Proteobacteria & $\gamma$-proteobacteria & Enterobacteriaceae & Raoultella & 0.38 & 11.09 & 0 \\
\hline Actinobacteria & Actinobacteria & Corynebacteriaceae & Corynebacterium & 0.005 & 8.76 & 1.17 \\
\hline Proteobacteria & $\gamma$-proteobacteria & Enterobacteriaceae & Enterobacter & 2.60 & 7.59 & 0.04 \\
\hline Proteobacteria & $\gamma$-proteobacteria & Enterobacteriaceae & Yersinia & 0.14 & 1.64 & 0.01 \\
\hline Actinobacteria & Actinobacteria & Coriobacteriaceae & Gordonibacter & 0.02 & 1.25 & 0.98 \\
\hline Proteobacteria & $\gamma$-proteobacteria & Xanthomonadaceae & Stenotrophomonas & 0.92 & 0.99 & 0.42 \\
\hline Proteobacteria & $\alpha$-proteobacteria & Rhodobacteraceae & Paracoccus & 0.01 & 0.01 & 30.94 \\
\hline Proteobacteria & $\beta$-proteobacteria & Comamonadaceae & Variovorax & 0.005 & 0.004 & 10.61 \\
\hline Bacteroidetes & Bacteroidia & Porphyromonadaceae & Petrimonas & 0.20 & 2.00 & 7.37 \\
\hline Proteobacteria & $\beta$-proteobacteria & Comamonadaceae & Brachymonas & 0.16 & 0.18 & 6.18 \\
\hline Bacteroidetes & Flavobacteria & Flavobacteriaceae & Chryseobacterium & 2.51 & 0.02 & 5.04 \\
\hline Bacteroidetes & Bacteroidia & Porphyromonadaceae & Proteiniphilum & 0.12 & 1.34 & 3.02 \\
\hline Proteobacteria & $\delta$-proteobacteria & Desulfovibrionaceae & Desulfovibrio & 0.26 & 0.74 & 2.39 \\
\hline Proteobacteria & $\beta$-proteobacteria & Rhodocyclaceae & Azonexus & 0 & 0 & 2.24 \\
\hline Proteobacteria & $\alpha$-proteobacteria & Brucellaceae & Ochrobactrum & 0.174 & 0.072 & 2.05 \\
\hline Proteobacteria & $\alpha$-proteobacteria & Bradyrhizobiaceae & Rhodopseudomonas & 0.01 & 0.27 & 1.31 \\
\hline Proteobacteria & $\beta$-proteobacteria & Alcaligenaceae & Alcaligenes & 0.24 & 0.12 & 1.26 \\
\hline Firmicutes & Clostridia & Clostridiales & Anaerovorax & 0 & 0.15 & 1.25 \\
\hline Proteobacteria & $\alpha$-proteobacteria & Phyllobacteriaceae & Aquamicrobium & 0.81 & 0.01 & 1.21 \\
\hline Proteobacteria & $\alpha$-proteobacteria & Brucellaceae & Brucella & 0.18 & 0.14 & 1.09 \\
\hline
\end{tabular}

Myroides and Dysgonomonas had appeared in the nitroaromatics-reducing biocathode but at few abundance (Liang et al., 2013; Wang et al., 2011). These reasons together maybe explain why they dominated in the initial inoculum.

In the gBC, Enterococcus (38.42\%), Psychrobacter (18.05\%), Raoultella (11.09\%), Corynebacterium (8.76\%) and Enterobacter (7.59\%) had obviously higher relative abundance than those in the initial inoculum or bBC. In the bBC, Paracoccus was obviously enriched (30.94\%), however which showed fewer relative abundance in the initial inoculum (0.01\%) and gBC (0.01\%). Previous study indicated that Paracoccus were more abundant on the denitrification biocathode biofilm portions in close proximity to the electrode suggested that it had advantage for the extracellular electrons transfer to the autotrophic denitrification reaction (Virdis et al., 2011). Variovorax (10.61\%), Petrimonas (7.37\%), Brachymonas (6.18\%), Chryseobacterium (5.04\%) and others (including nine genera less than $4 \%$ relative abundance respectively) were also enriched in the bBC. Petrimonas was found in the bioanode (0.51-2\%) (Lu et al., 2012; Zhang et al., 2012) but was not reported in the biocathode previously. Comamonas (2.44\%) and Pseudomonas (1.72\%) were relatively enriched in the bBC compared to those in the gBC despite of they dominated in the initial inoculum (Table 2). Pseudomonas could denitrify with an electrode as the sole electrons donor (Su et al., 2012; Virdis et al., 2011).
The potential function of these dominant genera mentioned above was revealed as followed. Firstly, most of the dominant genera (Escherichia, Pseudomonas, Mycobacterium, Flavobacterium, Raoultella, Enterobacter and Desulfovibrio) were capable of reducing nitroaromatics to corresponding aromatic amines or mineralization of nitroraomatics (Comamonas, Pseudomonas and Alcaligenes) (Claus et al., 2006; Ma et al., 2007; Marvin-Sikkema and de Bont, 1994; Spain, 1995) regardless of the performance mode. Subsequently, most of the dominant genera (Pseudomonas, Escherichia, Rhodopseudomonas, Ochrobactrum, Desulfovibrio, Enterobacter, Comamonas and Corynebacterium) were identified with electrochemically activity (Logan, 2009; Rosenbaum et al., 2011; Xing et al., 2010) or dominated in the biocathode (Enterococcus, Pseudomonas and Paracoccus) (Virdis et al., 2011; Wang et al., 2011) regardless of some of them dominated in the initial inoculum. Finally, some of dominant genera from autotroph were enriched in the $b B C$ and some of dominant genera from heterotroph were abundant in the initial inoculum or gBC (both with glucose supply). For example, Dysgonomonas and Escherichia could ferment glucose with production of volatile acid under facultative anaerobic conditions were enriched in the initial inoculum. Enterococcus (38.42\%) and Raoultella (11.09\%) dominated in the gBC attributed to they could reduce nitroaromatics into corresponding aromatic amines under heterotrophic cultivation (Claus et al., 2006; Rafii et al., 2003). 


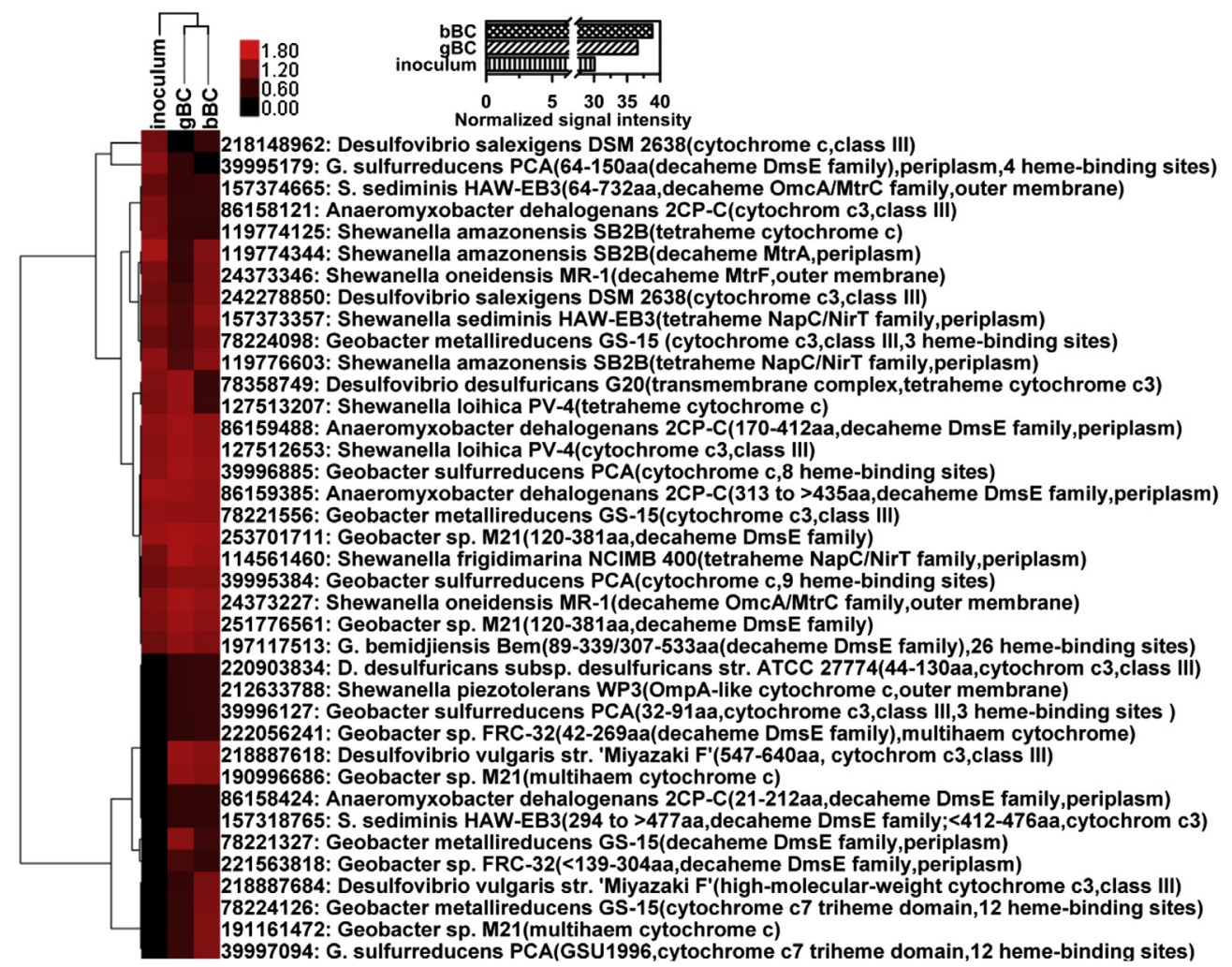

Fig. 7 - Hierarchical clustering of multiheme cytochrome c genes. Gene number (the protein ID number) and the annotation for each gene is obtained from the NCBI database. The inset histogram indicated the total abundance of cytochromes $c$.

\subsection{Carbon fixation genes suggest autotrophic energy conservation}

Genome sequencing of Variovorax paradoxus S110 indicated that it carried corresponding genes for $\mathrm{CO}_{2}$ fixation (Han et al., 2010). Moreover, GeoChip analysis detected three carbon fixation genes (Gene ID 220718833 and 221082971 for propionylCoA carboxylase and 239801525 for ribulose-bisphosphate carboxylase) from V. paradoxus S110 and Paracoccus denitrificans PD1222 (Gene ID 69936694 and 119386345 for propionyl-CoA carboxylase and 69153892 for ribulosebisphosphate carboxylase) respectively, which further supported the function of Variovorax and Paracoccus in the autotrophic bBC. Based on the GeoChip analysis, a total of 592 carbon fixation genes involved in the reductive pentose phosphate cycle, reductive citric acid cycle, reductive acetylCoA (Wood-Ljungdahl, WL) pathway, 3-hydroxypropionate bicycle and 3-hydroxypropionate-4-hydroxybutyrate cycle (Berg et al., 2010; Fuchs, 2011) were detected in this study (Fig. S1). The WL pathway is the most efficient, autotrophic carbon fixation pathway that can be coupled with energy conservation (Lovley and Nevin, 2013). A total of $126 \mathrm{CODH}$ genes that involved in WL pathway were detected and showed relatively higher abundance in the $b B C$ than in the $g B C$ (Fig. S1), suggesting that the bBC species may uptake electrons from the electrode and use these electrons to generate ATP and then produce biomass under autotrophic condition. Additionally, Variovorax was capable of mineralizing of aromatic amine 3,4-dichloroaniline to Krebs cycle intermediates
(Bers et al., 2011), suggesting that Variovorax of the bBC likely involved in the mineralization of aniline to support cathodophilic bacteria growth to a certain extent as the autotrophic $b B C$ generated significantly lower aniline than that of the heterotrophic gBC (Fig. 2A).

\subsection{C-type cytochrome genes suggest direct electron transfer}

In this study, a total of 211 cytochrome $c$ genes from six typical electrochemically active genera (Geobacter, Anaeromyxobacter, Shewanella, Pseudomonas, Desulfovibrio and Rhodobacter) (Hasan et al., 2012; Logan, 2009; Lovley, 2011; Rosenbaum et al., 2011) were detected. Fifty-five cytochrome $c$ genes undetected in the initial inoculum were enriched in the biocathode. Cytochromes $\mathrm{c}$ belonging to the class III family are distinguished by their multi-heme nature and negative redox potential (-160 to $-500 \mathrm{mV}$ ) (Bianco and Haladjian, 1994). Twelve class III cytochrome $c$ genes were detected, and another 26 having multiheme were also found. The total signal intensities for multiheme cytochrome $c$ genes in the gBC and bBC increased by 17.8 and $22.5 \%$, respectively, compared to that of the initial inoculum (Fig. 7). Two dodecaheme cytochrome c7 (represent a novel family) genes of Geobacter were only detected in the biocathode communities. Multiheme cytochrome c7 had a nanowire-like arrangement of 12 hemes might act as a capacitor and play an essential role in extracellular electrons transport processes (Pokkuluri et al., 2011). Relatively more 
multiheme cytochrome $c$ genes in the autotrophic bBC were likely involved in the maintenance of electrochemical activity.

Cytochrome c proteins are essential for electrons transfer in dissimilatory anaerobic respiration of extracellular electrons acceptors and could also reverse the electrons transfer pathway for intracellular reductive metabolism (Lovley, 2012; Richter et al., 2012a; Ross et al., 2011). In this study, the detected outer membrane cytochrome $c$ genes included decaheme OmcA/MtrC (Richter et al., 2012a), MtrF (a homologue of OmcA/MtrC) (Coursolle and Gralnick, 2010), and OmpA. Periplasmic multi-heme cytochrome $c$ including one decaheme MtrA, 11 decaheme DmsE (a homologue of MtrA) (Coursolle and Gralnick, 2010), 3 inner membrane anchored (periplasm side) tetraheme NapC/NirT (a homologue of quinol dehydrogenase CymA) (McMillan et al., 2012) and 2 transmembrane high-molecular-weight cytochrome electron-transferring complexes were detected (Fig. 7).

The diversity of cytochrome $c$ genes found on the biocathode likely attributable to the redox potential (RP) window of those cytochromes, which form a complete redox gradient-driven electrons transfer chain to deliver electrons from the biocathode interface to intracellular terminal reductases. At neutral $\mathrm{pH}$, the RP window of MtrC/OmcA, MtrF, MtrA and CymA are -0.5 to $0.1 \mathrm{~V},-0.4$ to $0.1 \mathrm{~V},-0.3$ to $0 \mathrm{~V}$ and -0.4 to $0 \mathrm{~V}$, respectively (Clarke et al., 2011; Firer-Sherwood et al., 2008; McMillan et al., 2012; Ross et al., 2011). Supply of the periplasmic electrons acceptor fumarate to a Shewanella oneidensis MR-1 formed biocathode (poised at $-0.36 \mathrm{~V}$ ) immediately led to electrons uptake for fumarate reduction, while a mutant lacking MtrA, MtrB or the menaquinone-linked CymA displayed a severe reduction deficiency, which showed that electrons primarily flowed from outer membrane cytochromes into the menaquinone pool, and then back to periplasmic fumarate reductase (Ross et al., 2011). Another study indicated that CymA of S. oneidensis MR-1 was important in electrons transfer for anaerobic NB reduction as the CymA knockout led to a $67 \%$ loss of $\mathrm{Er}_{\mathrm{NB}}$ with lactate as the electrons donor compared to the wild type (Cai et al., 2012). Three homologues of CymA belonging to the tetraheme NapC/NirT family showing high amino acid similarity (73.2-81.2\%) were also detected (Fig. 7).

\subsection{Pilin genes suggest cathode biofilm formation and potential conductivity}

A total of 244 Type IV pilins belonging to 151 species and four uncultured bacteria were detected. They were highly diverse as shown in Fig. S2. The phylogenetic tree was divided into five clusters including e.g., PilA, PilE, MshABCD and some unannotated pilin from 84 genera. Among these, 15 PilA sequences shared the highest amino acid similarity (42.5-57.9\%) to that in Geobacter sulfurreducens (39996596). PilA of G. sulfurreducens (39996596) and G. metallireducens (23053459) have the property of electro-conductivity that has been extensively studied (Malvankar et al., 2011; Reguera et al., 2005). Aromatic amino acids in the carboxyl terminus of PilA in G. sulfurreducens contributed to pilin conductivity and long-range extracellular electrons transport (Vargas et al., 2013). The biocathode had a higher total abundance and putative electro-conductive pilin genes (Msh and PilA) (Fitzgerald et al., 2012; Gorby et al., 2006; Malvankar et al., 2011) than those of the initial inoculum, while the $\mathrm{gBC}$ was enriched in pilin genes compared to the bBC (Fig. 8). A little bit of nitrosobenzene (0.02 mM at 24th h) was detected in the bBC but not in the gBC. The detected nitrosobenzene maybe produced through abiotic electrochemical NB reduction on the cathode surface without biofilm cover, which likely attributed to autotrophic cultivation yield less biocathode biofilm biomass than heterotrophic cultivation (Jeremiasse et al., 2012) and was also consistent with less extracellular pilin gene abundance in the bBC. Glucose was the intracellular electrons donor and growth carbon source for cathodophilic species of the gBC. The energy yield for the autotrophic bBC was low as cathodophilic species conserve energy only via carbon fixation.

Type IV pilin (extracellular nanofilaments) are versatile molecular modules that have been found in phylogenetically diverse bacteria (Giltner et al., 2012). The extracellular pilin networks had a key role in the biofilm formation and adherence to solid surfaces (Giltner et al., 2012). Type IV pilin are conductive and may be involved in direct (or associated with outermembrane cytochrome c) extracellular electrons transfer (Gorby et al., 2006; Malvankar et al., 2011) and play some role in cytochrome secretion (Richter et al., 2012b). Microbial nanowires could transfer electrons under certain environmental conditions such as limited electrons acceptor availability, Fe(II) oxides (similar to an electrode) providing electrons mode, presence of electrons accepting metals or an insoluble electrode as an electron acceptor (Fitzgerald et al., 2012; Gorby et al., 2006; Li and Li, 2013; Pfeffer et al., 2012; Reguera et al., 2005). Hence, the detected pilin genes from diverse bacteria in this study could contribute to the biocathode formation and maybe also involved in the extracellular electrons transfer during bioelectrochemical NB reduction. However, to confirm this analysis of the conductivity of pilin from diverse electrochemically active species of biocathode is needed.

\subsection{Diverse functional genes related to nitroaromatics reduction}

Members of the nitroreductase family can utilize FMN as a cofactor and catalyze reduction of a variety of nitroaromatic

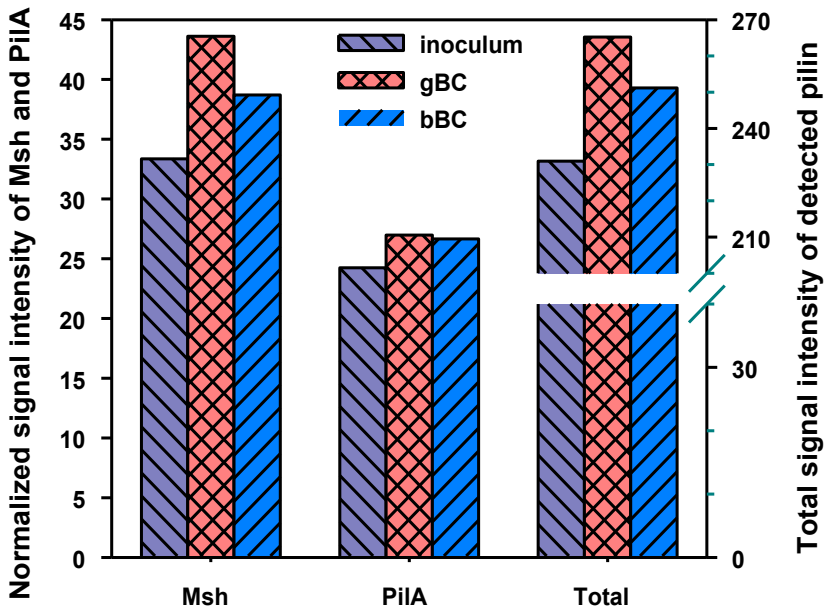

Fig. 8 - The normalized signal intensity of detected total and putative electro-conductive pilin genes (Msh and PilA) in the initial inoculum, gBC and bBC. 


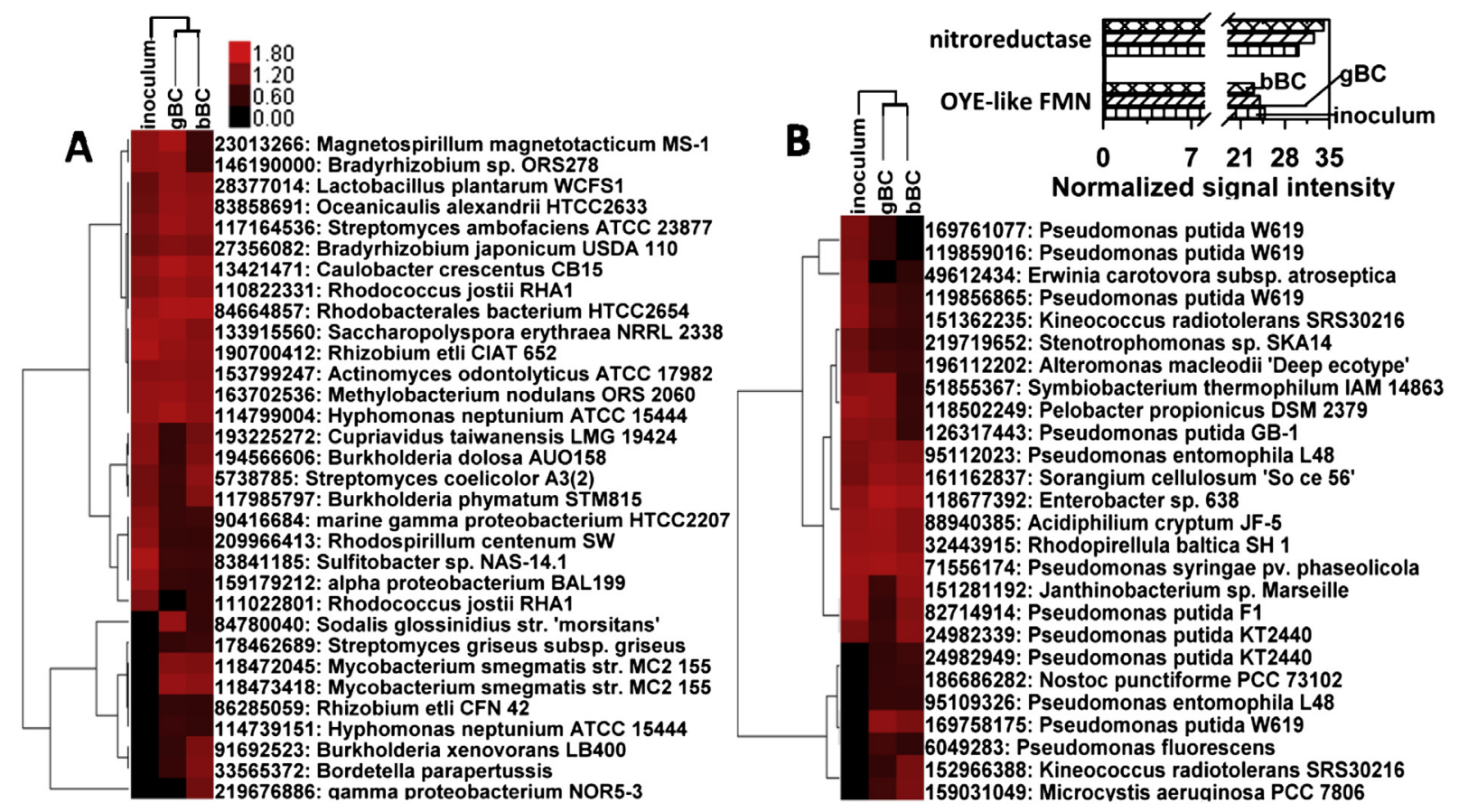

Fig. 9 - Hierarchical clustering of nitroreductase (A) and OYE-like FMN (B) genes. The inset histogram indicated the total abundance for each gene family.

compounds using $\mathrm{NAD}(\mathrm{P}) \mathrm{H}$ as electrons donor (Koder et al., 2002). In this study, 32 nitroreductase genes were detected that came from 19 genera and 3 uncultured proteobacteria ( $\alpha$ and $\gamma$ ) among 5 classes of Actinobacteria, Bacilli, $\alpha$-proteobacteria, $\beta$-proteobacteria and $\gamma$-proteobacteria. The initial inoculum had relatively low nitroreductase gene abundance. Nine nitroreductase genes undetected in the initial inoculum were enriched in the biocathode (Fig. 9A).

26 OYE-like FMN genes were detected, which belonged to 14 genera (Fig. 9B) among 6 classes of Actinobacteria, Cyanobacteria (phylum), Clostridia, Planctomycetacia, $\beta$-proteobacteria, $\delta$-proteobacteria and $\gamma$-proteobacteria. The OYE-like FMN was the first flavin-dependent enzyme that displayed nitroreductase activity (Yanto et al., 2010). Each monomer of OYE contains FMN as a non-covalently bound cofactor and uses NADPH as electrons donor. Seven OYE genes undetected in the initial

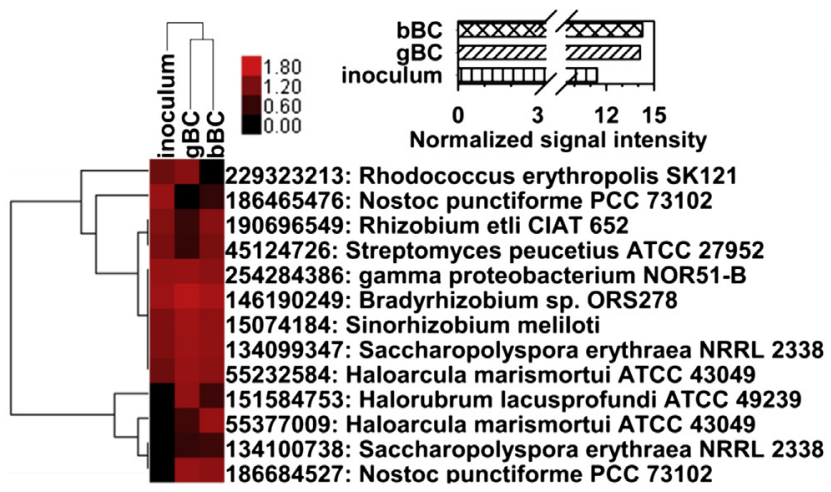

Fig. 10 - Hierarchical clustering of cytochrome P450 genes. The inset histogram indicated the total abundance of cytochrome P450. inoculum were enriched in the biocathode (Fig. 9B). The measured midpoint potential of the FMN cofactor of Enterobacter cloacae nitroreductase was -0.19 V (Koder et al., 2002), consistent with our observation that NB reduction with the gBC was found to start at around $-0.20 \mathrm{~V}$ (Fig. 2B).

Cytochrome $\mathrm{P} 450$ reductase has electrons transfer ability (Sevrioukova et al., 1999) and also shows nitroreductase activity (Fann et al., 1999). Thirteen cytochrome $\mathrm{P} 450$ genes from 9 genera and one uncultured $\gamma$-proteobacterium were detected. The abundance of cytochrome $\mathrm{P} 450$ genes was increased by $19.5 \%$ in the biocathode, suggesting that these genes may be involved in electrons transfer for NB reduction (Fig. 10).

\section{Conclusions}

Selective transformation of nitrobenzene to aniline maintained with biocathode communities after carbon source switchover. Continuous electrical field stimulation and carbon source switchover had markedly influences on the microbial community succession. Most of the dominant genera found in this study were capable of reducing nitroaromatics to the corresponding aromatic amines. Heterotrophic microbes dominated in the glucose-fed biocathode and that was replaced by autotrophic microbes when switching glucose to bicarbonate. Diverse multi-heme cytochrome $c$ and carbon fixation genes showed relatively higher abundances in the bicarbonate-fed biocathode, likely met the requirement of the energy conservation and maintained the nitrobenzene selective reduction capability. Diverse pilin genes was enriched in the glucose-fed biocathode might explain the enhancement of electrocatalysis activity for nitrobenzene reduction with glucose supply. The results presented in this study revealed the shift in the 
structure, richness and composition of microbial communities on nitrobenzene reduction biocathode in response to the carbon source switchover from both phylogenetics and functional gene perspectives and also suggested the potential mechanisms of the biocathode communities maintained the nitrobenzene reduction capability under varied carbon source supply conditions.

\section{Acknowledgment}

This research was supported by the National Natural Science Foundation of China (51078100 and 51176037), National Science Foundation for Distinguished Young Scholars (51225802), National Creative Research Groups Project (51121062), the Ph.D. Programs Foundation of Ministry of Education of China (20102302110055), and the State Key Laboratory of Urban Water Resource and Environment of HIT (2013DX02). Bin Liang's collaborative research at the Institute for Environmental Genomics of University of Oklahoma, was supported by the China Scholarship Council.

\section{Appendix A. Supplementary data}

Supplementary data related to this article can be found at http://dx.doi.org/10.1016/j.watres.2014.01.052.

\section{REFEREN C E S}

Berg, I.A., Kockelkorn, D., Ramos-Vera, W.H., Say, R.F., Zarzycki, J., Hugler, M., Alber, B.E., Fuchs, G., 2010. Autotrophic carbon fixation in archaea. Nat. Rev. Microbiol. 8 (6), 447-460.

Bers, K., Leroy, B., Breugelmans, P., Albers, P., Lavigne, R., Sorensen, S.R., Aamand, J., De Mot, R., Wattiez, R., Springael, D., 2011. A novel hydrolase identified by genomicproteomic analysis of phenylurea herbicide mineralization by Variovorax sp. strain SRS16. Appl. Environ. Microbiol. 77 (24), 8754-8764.

Bianco, P., Haladjian, J., 1994. Recent progress in the electrochemistry of c-type cytochromes. Biochimie 76 (7), 605-613.

Cai, P.J., Xiao, X., He, Y.R., Li, W.W., Yu, L., Lam, M.H.W., Yu, H.Q., 2012. Involvement of c-type cytochrome CymA in the electron transfer of anaerobic nitrobenzene reduction by Shewanella oneidensis MR-1. Biochem. Eng. J. 68, 227-230.

Caporaso, J.G., Lauber, C.L., Walters, W.A., Berg-Lyons, D., Huntley, J., Fierer, N., Owens, S.M., Betley, J., Fraser, L., Bauer, M., Gormley, N., Gilbert, J.A., Smith, G., Knight, R., 2012. Ultra-high-throughput microbial community analysis on the Illumina HiSeq and MiSeq platforms. Isme J. 6 (8), 1621-1624.

Clarke, T.A., Edwards, M.J., Gates, A.J., Hall, A., White, G.F., Bradley, J., Reardon, C.L., Shi, L., Beliaev, A.S., Marshall, M.J., Wang, Z.M., Watmough, N.J., Fredrickson, J.K., Zachara, J.M., Butt, J.N., Richardson, D.J., 2011. Structure of a bacterial cell surface decaheme electron conduit. Proc. Natl. Acad. Sci. USA 108 (23), 9384-9389.

Claus, H., Perret, N., Bausinger, T., Fels, G., Preuß, J., König, H., 2006. TNT transformation products are affected by the growth conditions of Raoultella terrigena. Biotechnol. Lett. 29 (3), 411-419.

Coursolle, D., Gralnick, J.A., 2010. Modularity of the Mtr respiratory pathway of Shewanella oneidensis strain MR-1. Mol. Microbiol. 77 (4), 995-1008.

Fann, Y.C., Metosh-Dickey, C.A., Winston, G.W., Sygula, A., Rao, D.N.R., Kadiiska, M.B., Mason, R.P., 1999. Enzymatic and nonenzymatic production of free radicals from the carcinogens 4-nitroquinoline $\mathrm{N}$-oxide and 4hydroxylaminoquinoline N-oxide. Chem. Res. Toxicol. 12 (5), 450-458.

Firer-Sherwood, M., Pulcu, G.S., Elliott, S.J., 2008. Electrochemical interrogations of the Mtr cytochromes from Shewanella: opening a potential window. J. Biol. Inorg. Chem. 13 (6), 849-854.

Fitzgerald, L.A., Petersen, E.R., Ray, R.I., Little, B.J., Cooper, C.J., Howard, E.C., Ringeisen, B.R., Biffinger, J.C., 2012. Shewanella oneidensis MR-1 Msh pilin proteins are involved in extracellular electron transfer in microbial fuel cells. Process Biochem. 47 (1), 170-174.

Fuchs, G., 2011. Alternative pathways of carbon dioxide fixation: Insights into the early evolution of life? Annu. Rev. Microbiol. $65,631-658$.

Giltner, C.L., Nguyen, Y., Burrows, L.L., 2012. Type IV Pilin proteins: versatile molecular modules. Microbiol. Mol. Biol. Rev. 76 (4), 740-772.

Gorby, Y.A., Yanina, S., McLean, J.S., Rosso, K.M., Moyles, D., Dohnalkova, A., Beveridge, T.J., Chang, I.S., Kim, B.H., Kim, K.S., Culley, D.E., Reed, S.B., Romine, M.F., Saffarini, D.A., Hill, E.A., Shi, L., Elias, D.A., Kennedy, D.W., Pinchuk, G., Watanabe, K., Ishii, S., Logan, B., Nealson, K.H.,

Fredrickson, J.K., 2006. Electrically conductive bacterial nanowires produced by Shewanella oneidensis strain MR-1 and other microorganisms. Proc. Natl. Acad. Sci. USA 103 (30), $11358-11363$.

Han, J.I., Choi, H.K., Lee, S.W., Orwin, P.M., Kim, J., LaRoe, S.L., Kim, T.g., O’Neil, J., Leadbetter, J.R., Lee, S.Y., Hur, C.G., Spain, J.C., Ovchinnikova, G., Goodwin, L., Han, C., 2010. Complete genome sequence of the metabolically versatile plant growth-promoting endophyte Variovorax paradoxus S110. J. Bacteriol. 193 (5), 1183-1190.

Hasan, K., Patil, S.A., Górecki, K., Leech, D., Hägerhäll, C., Gorton, L., 2012. Electrochemical communication between heterotrophically grown Rhodobacter capsulatus with electrodes mediated by an osmium redox polymer. Bioelectrochemistry 93, 30-36.

He, Z., Van Nostrand, J.D., Zhou, J., 2012. Applications of functional gene microarrays for profiling microbial communities. Curr. Opin. Biotechnol. 23 (3), 460-466.

Jeremiasse, A.W., Hamelers, H.V.M., Croese, E., Buisman, C.J.N., 2012. Acetate enhances startup of a $\mathrm{H}_{2}$-producing microbial biocathode. Biotechnol. Bioeng. 109 (3), 657-664.

Ju, K.S., Parales, R.E., 2010. Nitroaromatic compounds, from synthesis to biodegradation. Microbiol. Mol. Biol. Rev. 74 (2), 250-272.

Koder, R.L., Haynes, C.A., Rodgers, M.E., Rodgers, D.W., Miller, A.F., 2002. Flavin thermodynamics explain the oxygen insensitivity of enteric nitroreductases. Biochemistry 41 (48), 14197-14205.

Li, Y., Li, H., 2013. Type IV Pili of Acidithiobacillus ferrooxidans can transfer electrons from extracellular electron donors. J. Basic Microbiol. 53, 1-6.

Liang, B., Cheng, H.Y., Kong, D.Y., Gao, S.H., Sun, F., Cui, D., Kong, F.Y., Zhou, A.J., Liu, W.Z., Ren, N.Q., Wu, W.W., Wang, A.J., Lee, D.J., 2013. Accelerated reduction of chlorinated nitroaromatic antibiotic chloramphenicol by biocathode. Environ. Sci. Technol. 47 (10), 5353-5361. 
Liu, W., Wang, A., Cheng, S., Logan, B.E., Yu, H., Deng, Y., Nostrand, J.D., Wu, L., He, Z., Zhou, J., 2010. Geochip-based functional gene analysis of anodophilic communities in microbial electrolysis cells under different operational modes. Environ. Sci. Technol. 44 (19), 7729-7735.

Logan, B.E., 2009. Exoelectrogenic bacteria that power microbial fuel cells. Nat. Rev. Microbiol. 7 (5), 375-381.

Lovley, D.R., 2011. Powering microbes with electricity: direct electron transfer from electrodes to microbes. Environ. Microbiol. Reports 3 (1), 27-35.

Lovley, D.R., 2012. Electromicrobiology. Annu. Rev. Microbiol. 66, 391-409.

Lovley, D.R., Nevin, K.P., 2013. Electrobiocommodities: powering microbial production of fuels and commodity chemicals from carbon dioxide with electricity. Curr. Opin. Biotechnol. 24 (3), 385-390.

Lu, L., Xing, D.F., Ren, N.Q., 2012. Pyrosequencing reveals highly diverse microbial communities in microbial electrolysis cells involved in enhanced $\mathrm{H}_{2}$ production from waste activated sludge. Water Res. 46 (7), 2425-2434.

Ma, Y.F., Wu, J.F., Wang, S.Y., Jiang, C.Y., Zhang, Y., Qi, S.W., Liu, L., Zhao, G.P., Liu, S.J., 2007. Nucleotide sequence of plasmid pCNB1 from Comamonas strain CNB-1 reveals novel genetic organization and evolution for 4-chloronitrobenzene degradation. Appl. Environ. Microbiol. 73 (14), 4477-4483.

Malvankar, N.S., Vargas, M., Nevin, K.P., Franks, A.E., Leang, C., Kim, B.C., Inoue, K., Mester, T., Covalla, S.F., Johnson, J.P., Rotello, V.M., Tuominen, M.T., Lovley, D.R., 2011. Tunable metallic-like conductivity in microbial nanowire networks. Nat. Nanotechnol. 6 (9), 573-579.

Marvin-Sikkema, F.D., de Bont, J.A., 1994. Degradation of nitroaromatic compounds by microorganisms. Appl. Microbiol. Biotechnol. 42 (4), 499-507.

McMillan, D.G.G., Marritt, S.J., Butt, J.N., Jeuken, L.J.C., 2012. Menaquinone-7 is specific cofactor in tetraheme quinol dehydrogenase CymA. J. Biol. Chem. 287 (17), 14215-14225.

Mu, Y., Rozendal, R.A., Rabaey, K., Keller, J., 2009. Nitrobenzene removal in bioelectrochemical systems. Environ. Sci. Technol. 43 (22), 8690-8695.

Peres, C.M., Naveau, H., Agathos, S.N., 1998. Biodegradation of nitrobenzene by its simultaneous reduction into aniline and mineralization of the aniline formed. Appl. Microbiol. Biotechnol. 49 (3), 343-349.

Pfeffer, C., Larsen, S., Song, J., Dong, M.D., Besenbacher, F., Meyer, R.L., Kjeldsen, K.U., Schreiber, L., Gorby, Y.A., ElNaggar, M.Y., Leung, K.M., Schramm, A., RisgaardPetersen, N., Nielsen, L.P., 2012. Filamentous bacteria transport electrons over centimetre distances. Nature 491 (7423), 218-221.

Pokkuluri, P.R., Londer, Y.Y., Duke, N.E.C., Pessanha, M., Yang, X., Orshonsky, V., Orshonsky, L., Erickson, J., Zagyanskiy, Y., Salgueiro, C.A., Schiffer, M., 2011. Structure of a novel dodecaheme cytochrome c from Geobacter sulfurreducens reveals an extended $12 \mathrm{~nm}$ protein with interacting hemes. J. Struct. Biol. 174 (1), 223-233.

Rafii, F., Wynne, R., Heinze, T.M., Paine, D.D., 2003. Mechanism of metronidazole-resistance by isolates of nitroreductaseproducing Enterococcus gallinarum and Enterococcus casseliflavus from the human intestinal tract. FEMS Microbiol. Lett. 225 (2), 195-200.

Reguera, G., McCarthy, K.D., Mehta, T., Nicoll, J.S., Tuominen, M.T., Lovley, D.R., 2005. Extracellular electron transfer via microbial nanowires. Nature 435 (7045), 1098-1101.

Richter, K., Schicklberger, M., Gescher, J., 2012a. Dissimilatory reduction of extracellular electron acceptors in anaerobic respiration. Appl. Environ. Microbiol. 78 (4), 913-921.

Richter, L.V., Sandler, S.J., Weis, R.M., 2012b. Two isoforms of Geobacter sulfurreducens PilA have distinct roles in pilus biogenesis, cytochrome localization, extracellular electron transfer, and biofilm formation. J. Bacteriol. 194 (10), 2551-2563.

Roldan, M.D., Perez-Reinado, E., Castillo, F., Moreno-Vivian, C., 2008. Reduction of polynitroaromatic compounds: the bacterial nitroreductases. FEMS Microbiol. Rev. 32 (3), 474-500.

Rosenbaum, M., Aulenta, F., Villano, M., Angenent, L.T., 2011. Cathodes as electron donors for microbial metabolism: which extracellular electron transfer mechanisms are involved? Bioresour. Technol. 102 (1), 324-333.

Ross, D.E., Flynn, J.M., Baron, D.B., Gralnick, J.A., Bond, D.R., 2011. Towards electrosynthesis in Shewanella: energetics of reversing the Mtr pathway for reductive metabolism. PLoS One 6 (2), e16649.

Sevrioukova, I.F., Li, H.Y., Zhang, H., Peterson, J.A., Poulos, T.L., 1999. Structure of a cytochrome P450-redox partner electrontransfer complex. Proc. Natl. Acad. Sci. USA 96 (5), 1863-1868.

Shen, J., Zhang, Y., Xu, X., Hua, C., Sun, X., Li, J., Mu, Y., Wang, L., 2013. Role of molecular structure on bioelectrochemical reduction of mononitrophenols from wastewater. Water Res. 47 (15), 5511-5519.

Spain, J.C., 1995. Biodegradation of nitroaromatic compounds. Annu. Rev. Microbiol. 49, 523-555.

Su, W., Zhang, L., Li, D., Zhan, G., Qian, J., Tao, Y., 2012. Dissimilatory nitrate reduction by Pseudomonas alcaliphila with an electrode as the sole electron donor. Biotechnol. Bioeng. 109 (11), 2904-2910.

Vargas, M., Malvankar, N.S., Tremblay, P.L., Leang, C., Smith, J.A., Patel, P., Synoeyenbos-West, O., Nevin, K.P., Lovley, D.R., 2013. Aromatic amino acids required for Pili conductivity and longrange extracellular electron transport in Geobacter sulfurreducens. mBio 4 (2), e00105-e00113.

Virdis, B., Read, S.T., Rabaey, K., Rozendal, R.A., Yuan, Z., Keller, J., 2011. Biofilm stratification during simultaneous nitrification and denitrification (SND) at a biocathode. Bioresour. Technol. 102 (1), 334-341.

Wang, A.J., Cheng, H.Y., Liang, B., Ren, N.Q., Cui, D., Lin, N., Kim, B.H., Rabaey, K., 2011. Efficient reduction of nitrobenzene to aniline with a biocatalyzed cathode. Environ. Sci. Technol. 45 (23), 10186-10193.

Wang, A.J., Cui, D., Cheng, H.Y., Guo, Y.Q., Kong, F.Y., Ren, N.Q., Wu, W.M., 2012. A membrane-free, continuously feeding, single chamber up-flow biocatalyzed electrolysis reactor for nitrobenzene reduction. J. Hazard. Mater. 199-200, 401-409.

Wrighton, K.C., Virdis, B., Clauwaert, P., Read, S.T., Daly, R.A., Boon, N., Piceno, Y., Andersen, G.L., Coates, J.D., Rabaey, K., 2010. Bacterial community structure corresponds to performance during cathodic nitrate reduction. Isme J. 4 (11), 1443-1455.

Xing, D.F., Cheng, S.A., Logan, B.E., Regan, J.M., 2010. Isolation of the exoelectrogenic denitrifying bacterium Comamonas denitrificans based on dilution to extinction. Appl. Microbiol. Biotechnol. 85 (5), 1575-1587.

Yanto, Y., Hall, M., Bommarius, A.S., 2010. Nitroreductase from Salmonella typhimurium: characterization and catalytic activity. Org. Biomol. Chem. 8 (8), 1826-1832.

Zhang, G.D., Zhao, Q.L., Jiao, Y., Wang, K., Lee, D.J., Ren, N.Q., 2012. Biocathode microbial fuel cell for efficient electricity recovery from dairy manure. Biosens. Bioelectron. 31 (1), 537-543.

Zhang, Y., Xie, J., Liu, M., Tian, Z., He, Z., van Nostrand, J.D., Ren, L., Zhou, J., Yang, M., 2013. Microbial community functional structure in response to antibiotics in pharmaceutical wastewater treatment systems. Water Res. 47 (16), 6298-6308.

Zhou, J., Liu, W., Deng, Y., Jiang, Y.H., Xue, K., He, Z., Van Nostrand, J.D., Wu, L., Yang, Y., Wang, A., 2013. Stochastic assembly leads to alternative communities with distinct functions in a bioreactor microbial community. mBio 4 (2), e00584-12. 